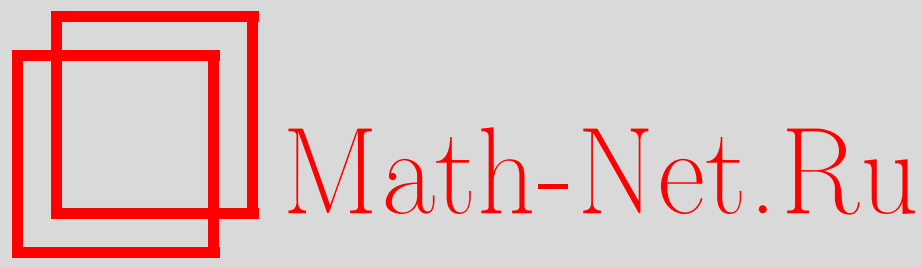

К. Гарола, С. Соццо, Представление и интерпретация квантовых смесей в модели развитого семантического реализма, ТМФ, 2011, том 168, номер 1, 65-79

DOI: https://doi.org/10.4213/tmf6664

Использование Общероссийского математического портала Math-Net.Ru подразумевает, что вы прочитали и согласны с пользовательским соглашением http://www . mathnet.ru/rus/agreement

Параметры загрузки:

IP: 34.229 .45 .116

26 апреля 2023 г., $14: 46: 41$

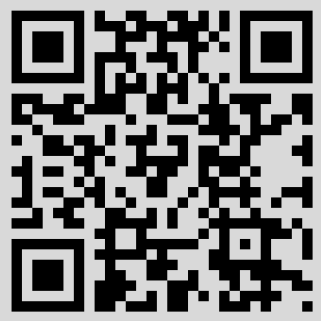




\title{
ФИЗИКА
}

Том 168, № 1

июль, 2011

\section{ПРЕДСТАВЛЕНИЕ И ИНТЕРПРЕТАЦИЯ КВАНТОВЫХ СМЕСЕЙ В МОДЕЛИ РАЗВИТОГО СЕМАНТИЧЕСКОГО РЕАЛИЗМА}

\begin{abstract}
Интерпретация смесей в квантовой механике в соответствии с этой теорией сталкивается с проблемами ввиду необъективности их физических свойств. В этой связи была разработана модель развитого семантического реализма, в которой происходит восстановление объективности в силу того, что квантовые вероятности интерпретируются при детектировании как условные, что позволяет ввести математический формализм квантовой механики в более общее неконтекстуальное (а потому локальное) рассмотрение. В этой модели каждая обобщенная наблюдаемая представляется в виде семейства положительных операторно-значных мер, параметризованных чистыми состояниями рассматриваемой физической системы $\Omega$. В настоящей работе предложено новое доказательство утверждения о том, что каждая собственная смесь задается семейством операторов плотности, параметризованных макроскопическими характеристиками системы $\Omega$. Показано, что некоторые предсказания, вытекающие из этого представления, отличаются от предсказаний квантовой механики, и они оказываются свободными от проблем, возникающих в стандартном представлении квантовой механики для собственных смесей. Напоминается, что преобразования состояний, вызванные идеальными неразрушающими измерениями, можно получить с помощью нетривиального обобщения постулата Людерса.
\end{abstract}

Ключевые слова: квантовая механика, квантовые смеси, квантовая вероятность, постулат Людерса.

\section{1. ВВЕДЕНИЕ}

Стандартная (копенгагенская) интерпретация квантовой механики (KM) предполагает, что свойства индивидуальной физической системы (физических объектов) в общем случае необъективны в том смысле, что свойство физического объекта, например значение наблюдаемой, не может быть представлено присущим или неприсущим объекту, пока не произведено измерение. Отсюда следует, в частности, что квантовые вероятности, вообще говоря, неэпистемичны, т. е. их невозможно интерпретировать формализующими субъективное неведение того, что происходит на

*Dipartimento di Fisica dell'Università del Salento, INFN-Sezione di Lecce, Lecce, Italy. E-mail: Garola@le.infn.it, Sozzo@le.infn.it 
микроскопическом уровне, как это имеет место в классической физике: наоборот, эти вероятности вскрывают внутреннюю неопределенность физического мира. Но само понятие необъективности лежит в основе концептуальных трудностей КМ, так как из него следуют известные парадоксы и проблема облективизации квантовой теории измерений, которая возникает сразу, как только допускается весьма слабая форма реализма (предположение о том, что КМ описывает индивидуальные объекты и их свойства), и этот парадокс так и остался неразрешенным в КМ и в ее нечетких расширениях [1]-[3].

Необъективность также играет роль в задачах, возникающих при попытке дать интерпретацию квантовым смесям. Понятие смеси вводится в стандартной формулировке КМ в терминах гильбертова пространства при учете физических ситуаций, в которых чистое состояние физической системы неизвестно досконально (см., например, [4], [5]). Если физическая система $\Omega$ приготовляется в таком виде, что с вероятностью $p_{1}$ она находится в чистом состоянии $S_{1}$, представленном проекционным оператором $\left|\psi_{1}\right\rangle\left\langle\psi_{1}\right|$, а с вероятностью $p_{2}$ - в чистом состоянии $S_{2}$, представленном проекционным оператором $\left|\psi_{2}\right\rangle\left\langle\psi_{2}\right|$, и т. д, то можно утверждать, что состояние $\Omega$ оказывается смесью $S$, и представляет $S$ с помощью оператора плотности $\rho_{S}=\sum_{j} p_{j}\left|\psi_{j}\right\rangle\left\langle\psi_{j}\right|$. Вероятность $p_{j}$ эпистемична в том смысле, что она выражает субъективное отсутствие знания настоящего состояния $\Omega$ и, следовательно, отсутствие априорного знания результата измерения с помощью оператора $\left|\psi_{j}\right\rangle\left\langle\psi_{j}\right|$, определяющего, присуще ли свойство $E_{j}$ объекту $\Omega$. Но при этом возникает проблема интерпретации. Действительно, оператор плотности $\rho_{S}$ допускает несколько (на самом деле бесконечно много) выпуклых разложений на чистые состояния, и коэффициенты, возникающие в разложениях, отличающихся от $\sum_{j} p_{j}\left|\psi_{j}\right\rangle\left\langle\psi_{j}\right|$, невозможно интерпретировать как эпистемические вероятности, поскольку эти коэффициенты суть комбинации эпистемических вероятностей $p_{1}, p_{2}, \ldots$ с неэпистемическими квантовыми вероятностями. Если задан один лишь оператор плотности $\rho_{S}$ в отсутствие дополнительной физической информации (что достаточно для вычисления всех необходимых вероятностей), то невозможно вычленить привилегированное разложение оператора $\rho_{S}$, коэффициенты которого можно интерпретировать как эпистемические вероятности. В связи с этим некоторые авторы считают, что интерпретация смесей в терминах незнания несостоятельна (см., например, [6]-[9]). Другие авторы, наоборот, считают, что концептуальные неоднозначности, возникающие из неоднозначной разложимости квантовых смесей, появляются за счет ограниченных возможностей технического языка описания KM, на котором невозможно выразить некоторые физически значимые особенности рассмотрения (см., например, [10], [11]).

Еще бо́льшие трудности возникают при описании составных систем. Действительно, если рассмотреть подсистему $\Omega_{1}$ составной системы $\Omega$, состоящей из подсистем $\Omega_{1}, \Omega_{2}, \ldots, \Omega_{n}$ в чисто запутанном состоянии, задаваемом оператором проекции $|\Psi\rangle\langle\Psi|$, то физическую информацию, обеспечиваемую КМ для подсистемы $\Omega_{1}$, можно получить с помощью вычисления частичного следа от величины $|\Psi\rangle\langle\Psi|$ относительно подсистем $\Omega_{2}, \ldots, \Omega_{n}$ с последующим применением стандартных правил квантовых вычислений к оператору $\rho_{1}$, полученному таким образом. Этот оператор плотности формально похож на оператор $\rho_{S}$. Но его коэффициенты никоим образом не могут быть интерпретированы как эпистемические вероятности, в отличие от коэффициентов оператора $\rho_{S}$, поскольку первые представляют собой неэпистемические квантовые вероятности. Из этого рассуждения, в частности, вытекает, что само 
соотнесение состояний к подсистемам составной физической системы оказывается плохо определенным в КМ. Поэтому некоторые авторы утверждают, что $\rho_{1}$ задает несобственную смесь, и вводят различие между такими смесями и собственными смесями, примером которых может служить $S$ (см., например, [1], [10], [11]). Другие авторы, наоборот, склонны критиковать сомнительный процесс коллапса, вводимого в рамках стандартной интерпретации проекционного постулата KM, и утверждают, что поскольку всякая физическая система создается в процессе взаимодействия с другой системой и потому представляет собой подсистему некоторой более объемлющей системы, собственные смеси не возникают в природе, и всякая смесь должна быть несобственной [7]-[9].

В силу указанных трудностей можно задаться вопросом о том, допускает ли интерпретация математического формализма КМ какие-либо уточнения, которые восстанавливают объективность свойств и позволяют интерпретировать все вероятности как эпистемические. Представляется, что эта возможность исключается известными запрещающими теоремами Белла [12] и Белла-Кошена-Спекера (БКС) [13], [14], которые нацелены на то, чтобы показать, что КМ обладает некоторыми характеристиками, а именно свойствами нелокальности и контекстуальности, из которых вытекает необъективность ее свойств. Поэтому любая попытка восстановления объективности должна основываться на предварительном критическом осмыслении этих теорем. В этой связи в наших предыдущих работах было показано, что доказательства теорем Белла и БКС основываются на эпистемологическом предположении, которое обычно остается незамеченным, но при этом вызывающим вопросы при рассмотрении с точки зрения квантовой науки (метатеоретический классический принцип (МКП)) [15]-[18]. Если заменить МКП более слабым предположением метатеоретического обобщенного приниипа (МОП), то доказательства указанных выше теорем не удается завершить и при этом можно построить интерпретацию математического формализма КМ в рамках семантического реализмa (CP), которая оказывается неконтекстуальной (а потому объективной и локальной $\left.{ }^{1)}\right)$. Более того, в работах [21]-[23] были разработаны разнообразные модели, показывающие самосогласованность интерпретации СР. Последняя из этих моделей модель развитого семантического реализма (PCP) - представляет собой новый тип теории неконтекстуальных скрытых параметров, в которой помимо скрытых переменных вводится новая интерпретация понятия квантовых вероятностей как условных относительно детектирования, а не абсолютных [24]-[27] ${ }^{2)}$. Модель РСР модифицирует и развивает интерпретацию, предложенную в модели СР, но наследует ее основное свойство, а именно подстановку МОП вместо МКП. Макроскопическая составляющая модели РСР может быть при этом сформулирована без обращения к скрытым переменным как самосогласованная теоретическая конструкция, переводящая математический формализм формулировки КМ в терминах гильбертова пространства в более широкие неконтекстуальные рамки, в которых дается новая

1)Нами также было доказано [19], что задача соотнесения состояний к подсистемам составной физической системы снимается с повестки дня в интерпретации СР. Добавим, что собственные и несобственные смеси можно сопоставить различным операторам плотности в кватернионной формулировке KM, что позволяет ввести различие между этими концептуально разными видами смесей также и с математической точки зрения в указанной формулировке [20].

2) Идея рассмотрения квантовых вероятностей как условных имеет долгую историю, краткий литературный обзор этого вопроса содержится в работе [28]. Но введение условности при детектировании, что и происходит в модели РСР, открывает новые возможности. 
интерпретация понятию квантовой вероятности и делаются предсказания, часть из которых отличается от предсказаний КМ [29]-[34].

В первых версиях модели РСР были получены вероятности для исходов обобщенных наблюдаемых и преобразований состояний, вызванных идеальными измерениями, при которых в расчет брались только чистые состояния [30]. Учет смесей требует предварительного уточнения того, имеется ли в модели РСР по-прежнему различие между собственными и несобственными смесями, а если имеется, то какой тип смеси рассматривается. Будет показано, что в модели РСР различие между собственными и несобственными смесями отсутствует, если обратиться к различиям между эпистемическими и неэпистемическими вероятностями, поскольку все вероятности оказываются эпистемическими в силу объективности свойств. Тем не менее имеют место эмпирические различия между двумя типами смесей, на основе которых можно их различать (см., например, [11]). Имея в виду это обстоятельство, авторам удалось распространить модель РСР на случай собственных смесей [32], [33], и дальнейшая задача состоит в углублении и улучшении нашего подхода с тем, чтобы показать, что обобщение модели РСР на случай собственных смесей не производится напрямую и приводит к некоторым физически значимым результатам ${ }^{3)}$. Краткий обзор предыдущих исследований приводится в разделе 2, где формализм модифицируется и приводится в соответствие с целями настоящей работы. В разделе 3 показано, что вычисление вероятностей исходов обобщенной наблюдаемой в случае собственной смеси требует наложения условия того, что смесь ассоциирована с семейством операторов плотности, параметризованных набором свойств рассматриваемой физической системы, а не просто с отдельным оператором плотности, как это имеет место в КМ. Важным физическим следствием оказывается то, что из этого нового представления вытекают предсказания для вероятностей, которые не совпадают с предсказаниями КМ и могут быть проверены эмпирически, по крайней мере в принципе. Более того, в разделе 4 показано, что представление собственной смеси зависит от физической процедуры, предпринятой при приготовлении этой смеси (операциональное определение собственной смеси), что позволяет избежать потери физической информации, что неизбежно происходит, если проводить рассмотрение в рамках стандартного подхода KM для собственных смесей. Наконец, в рамках нашего представления можно предложить нетривиальное обобщение постулата Людерса, что составляет содержание раздела 5.

\section{2. ОПИСАНИЕ МОДЕЛИ РСР В РАМКАХ ГИЛЬБЕРТОВА ПРОСТРАНСТВА}

Напомним основные свойства модели РСР и приведем некоторые из полученных ранее результатов [30], [32] в виде, более пригодном для целей настоящей работы.

В макроскопической части модели РСР физическая система $\Omega$ характеризуется соответствующими множествами $\mathcal{S}$ и $\mathcal{O}$ макроскопических состояний и макроскопических обобщенных наблюдаемых. Каждое состояние $S \in \mathcal{S}$ можно операционально определить как класс вероятностно эквивалентных приготовляющих устройств [8],

3) В данной работе не рассматриваются несобственные смеси, но следует отметить, что наше представление отличается от представления собственных смесей в соответствии с моделью РСР (что представляет собой замечательный результат, поскольку он позволяет устранить все несогласованности, связанные с физически различными представлениями), как будет показано в последующих публикациях. 
а каждое приготовляющее устройство $\pi \in S$ при активации приготавливает индивидуальный пример $x$ системы $\Omega$ (физический объект: можно коротко это определить, сказав, что в этом случае " $x$ находится в состоянии $S$ "). Каждая обобщенная наблюдаемая $A_{0} \in \mathcal{O}$ операционально определяется как класс вероятностно эквивалентных регистрирующих устройств, и она получается в модели $\mathrm{PCP}$, если рассмотреть KM-наблюдаемую $A$ вместе с набором возможных значений $\Xi$ на вещественной прямой $\mathbb{R}$ и добавить возможный исход $a_{0} \in \mathbb{R} \backslash \Xi$ (исход “отсутствие регистрации" $A_{0}$ ), так что множество всех возможных значений $A_{0}$ будет иметь вид ${ }^{4)} \Xi_{0}=\left\{a_{0}\right\} \cup \Xi$.

Более того, множество $\mathcal{F}_{0}$ всех (макроскопических) свойств системы $\Omega$ можно определить как

$$
\mathcal{F}_{0}=\left\{\left(A_{0}, X\right) \mid A_{0} \in \mathcal{O}, X \in \mathbb{B}(\mathbb{R})\right\},
$$

где $\mathbb{B}(\mathbb{R})$ - сигма-алгебра всех борелевских подмножеств прямой $\mathbb{R}$, а подмножество $\mathcal{F} \subset \mathcal{F}_{0}$ всех свойств, ассоциированных с КМ-наблюдаемой, можно задать выражением

$$
\mathcal{F}=\left\{\left(A_{0}, X\right) \mid A_{0} \in \mathcal{O}, X \in \mathbb{B}(\mathbb{R}), a_{0} \notin X\right\} .
$$

Измерение свойства $F=\left(A_{0}, X\right)$ на физическом объекте $x$ в состоянии $S$ при этом описывается как регистрация, производимая с помощью дихотомического регистрационного устройства (которое можно сконструировать, применяя одно из устройств, ассоциированных с наблюдаемой $A_{0}$ ), исходы которой обозначаются как “да" и "нет". Если измерение приводит к исходу “да" ("нет") (т.е. к исходу, в котором $x$ проявляет (не проявляет) свойство $F$ ), то значение наблюдаемой $A_{0}$ принадлежит (не принадлежит) множеству $X$. При $F=\left(A_{0}, X\right) \in \mathcal{F}$ (при этом $\left.a_{0} \notin X\right)$ полная вероятность того, что физический объект $x$ в состоянии $S$ проявляет свойство $F$, задается формулой

$$
p_{S}^{\mathrm{t}}(F)=p_{S}^{\mathrm{d}}(F) p_{S}(F)
$$

Символом $p_{S}^{\mathrm{d}}(F)$ в уравнении $(3)$ обозначена вероятность того, что физический объект $x$ будет детектирован, если он находится в состоянии $S$ (вероятность регистрации) и измеряется свойство $F$, и этот символ не обязательно фиксирован при заданном $A_{0}$ и может сам зависеть от свойства $F$ и принадлежать тем самым борелевскому множеству $X$. Предполагается, что существуют такие измерения, в которых $p_{S}^{\mathrm{d}}(F)$ зависит только от свойств физических объектов в состоянии $S$, потому этот символ не проявляется в силу дефектов или недостаточной эффективности аппаратуры, измеряющей свойство $F$, и рассматриваются только измерения такого рода $^{5)}$ (идеальные измерения). Символом $p_{S}(F)$ в уравнении (3) обозначена вероятность того, что $x$ демонстрирует свойство $F$ при регистрации. Тогда введем следующее

ПРЕДПОЛОЖЕНИЕ. Если $S$ - чистое состояние, то вероятность $p_{S}(F)$ можно вычислить, применяя те же правила, что и при построении вероятности свойства $F$ в состоянии $S$ в подходе KM.

\footnotetext{
4) Здесь предполагается для простоты, что множество $\mathbb{R} \backslash \Xi$ не пусто. Это предположение не налагает ограничений. В самом деле, если $\Xi=\mathbb{R}$, то можно выбрать биективную функцию Бореля $f: \mathbb{R} \rightarrow \Xi^{\prime}$ такую, что $\Xi^{\prime} \subset \mathbb{R}$ (например, $\Xi^{\prime}=\mathbb{R}^{+}$), и заменить $A$ на $f(A)$.

5)Это предположение можно подкрепить рассмотрением микроскопической составляющей модели РСР, которая не обсуждается в настоящей работе (см., в частности, работы [24], [26], [27]).
} 
Это предположение позволяет восстановить базисный формализм КМ в рамках модели РСР, но модифицирует стандартную интерпретацию квантовых вероятностей. В самом деле, в соответствии с КМ при произведении идеального измерения свойства $F$ регистрируются все физические объекты, приготовленные в состоянии $S$, а потому квантовые правила вычисления вероятностей интуитивно интерпретируются как правила, позволяющие вычислять вероятность того, что физический объект $x$ проявляет свойство $F$, как только он выбран из множества всех объектов, находящихся в состоянии $S$ (абсолютная вероятность). В соответствии с приведенным предположением, наоборот, если состояние $S$ чистое, то с помощью тех же правил можно получить вероятность проявления свойства $F$ физическим объектом $x$ в случае, когда этот объект выбирают из подмножества всех объектов в регистрируемом состоянии $S$ (условная вероятность).

Остается рассмотреть свойство $G=\left(A_{0}, Y\right) \in \mathcal{F}_{0} \backslash \mathcal{F}$ (следовательно, $\left.a_{0} \in Y\right)$. Полагая $X=Y \backslash\left\{a_{0}\right\}, F=\left(A_{0}, X\right)$ и $F^{c}(A, \mathbb{R} \backslash Y)$, мы вводим физически оправданное предположение

$$
p_{S}^{\mathrm{t}}(G)=1-p_{S}^{\mathrm{t}}\left(F^{c}\right)=1-p_{S}^{\mathrm{d}}\left(F^{c}\right) p_{S}\left(F^{c}\right)
$$

для каждого состояния $S$, которое описывает полную вероятность $p_{S}^{\mathrm{t}}(G)$ того, что физический объект $x$ в состоянии $S$ проявляет свойство $G \in \mathcal{F}_{0} \backslash \mathcal{F}$, когда $G$ измеряется в терминах полной вероятности того, что $x$ проявляет свойство $F \in \mathcal{F}^{c}$ при измерении $F^{c}$.

Чтобы задать вышеприведенные понятия в математическом виде, рассмотрим обобщенную наблюдаемую $A_{0}$, полученную из KM-наблюдаемой $A$, заданной самосопряженным оператором $\widehat{A}$. В работе [30] было доказано, что $A_{0}$ представимо в виде семейства коммутативных положительно-определенных операторно-значных (ПОЗ) мер

$$
\left\{T_{\psi}^{\widehat{A}}: X \in \mathbb{B}(\mathbb{R}) \longmapsto T_{\psi}^{\widehat{A}}(X) \in \mathscr{B}(\mathscr{H})\right\}_{|\psi\rangle \in \mathscr{V}},
$$

где $\mathscr{B}(\mathscr{H})$ - множество всех ограниченных операторов на $\mathscr{H}$, а $\mathscr{V}$ представляет собой множество всех единичных векторов пространства $\mathscr{H}$. Отображение $T_{\psi}^{\widehat{A}}$ определено как

$$
T_{\psi}^{\widehat{A}}(X)= \begin{cases}\int_{X} p_{\psi}^{\mathrm{d}}(\widehat{A}, \lambda) d P_{\lambda}^{\widehat{A}}, & a_{0} \notin X, \\ I-\int_{\mathbb{R} \backslash X} p_{\psi}^{\mathrm{d}}(\widehat{A}, \lambda) d P_{\lambda}^{\widehat{A}}, & a_{0} \in X,\end{cases}
$$

где $P^{\widehat{A}}$ - мера спектральной проекции, ассоциированная с $\widehat{A}$ и $p_{\psi}^{\mathrm{d}}(\widehat{A}, \lambda)$ и такая, что при каждом $|\psi\rangle \in \mathscr{V}$ величина $\left\langle\psi\left|p_{\psi}^{\mathrm{d}}(\widehat{A}, \lambda) \frac{d P_{\lambda}^{\widehat{A}}}{d \lambda}\right| \psi\right\rangle$ оказывается измеримой функцией на $\mathbb{R}$. Применяя это представление, можно вычислить вероятность $p_{S}^{\mathrm{t}}\left(\left(A_{0}, X\right)\right)$ того, что исход идеального измерения величины $A_{0}$ на физическом объекте $x$ в чистом состоянии $S$, представленный единичным вектором $|\psi\rangle$, лежит в борелевском множестве $X$ (или, эквивалентно, что вероятность измерения свойства $F=\left(A_{0}, X\right) \in \mathcal{F}_{0}$ 
на объекте $x$ приводит к положительному исходу6) (“да”)). Далее, можно обобщить проекционный постулат КМ и выписать вектор, задающий итоговое состояние после неразрушающего идеального измерения свойства $F=\left(A_{0}, X\right) \in \mathcal{F}_{0}$ на физическом объекте $x$. Представим теперь эти результаты, задающие $S$ в терминах операторов плотности $\rho_{\psi}=|\psi\rangle\langle\psi|$ вместо вектора $|\psi\rangle$. Во-первых, имеем

$$
p_{S}^{\mathrm{t}}\left(\left(A_{0}, X\right)\right)=\operatorname{Tr}\left[\rho_{\psi} T_{\psi}^{\widehat{A}}(X)\right] .
$$

Во-вторых, введем

ОБОБЩЕННЫЙ ПРОЕКЦИОННЫЙ ПОСТУЛАТ (ОПП). Пусть $S$ - чистое cocmoяние, представляемое оператором плотности $\rho_{\psi}$, и пусть над физическим обгектом $x$ в состоянии $S$ производится неразрушающее идеальное измерение физического свойства $F=\left(A_{0}, X\right) \in \mathcal{F}_{0}$.

Пусть исход измерения положителен. Тогда состояние $S_{F}$ обгекта $x$ после измерения является чистым состоянием, представляемым оператором плотности

$$
\rho_{\psi_{F}}=\frac{T_{\psi}^{\widehat{A}}(X) \rho_{\psi} T_{\psi}^{\widehat{A} \dagger}(X)}{\operatorname{Tr}\left[T_{\psi}^{\widehat{A}}(X) \rho_{\psi} T_{\psi}^{\widehat{A} \dagger}(X)\right]} .
$$

Пусть исход измерения отрицателен. Тогда состояние $S_{F}^{\prime}$ обгекта $x$ после измерения является чистым состоянием, представляемым оператором плотности

$$
\rho_{\psi_{F}^{\prime}}=\frac{T_{\psi}^{\widehat{A}}(\mathbb{R} \backslash X) \rho_{\psi} T_{\psi}^{\widehat{A} \dagger}(\mathbb{R} \backslash X)}{\operatorname{Tr}\left[T_{\psi}^{\widehat{A}}(\mathbb{R} \backslash X) \rho_{\psi} T_{\psi}^{\widehat{A} \dagger}(\mathbb{R} \backslash X)\right]} .
$$

Выражения (8) и (9) обобщают постулат Людерса в КМ, но это обобщение справедливо только для чистых состояний. Подчеркнем, что ОПП не предполагает никакой объективизации, поскольку все физические свойства объективны в модели РСР, как уже было объяснено в разделе 1.

Наконец, получим из приведенного выше предположения и соотношений (3) и (7), что при каждом $|\psi\rangle \in \mathscr{V}$ и $F=\left(A_{0}, X\right) \in \mathcal{F}$ (следовательно, при $\left.a_{0} \notin X\right)$ выполняется следующее соотношение:

$$
\operatorname{Tr}\left[\rho_{\psi} T_{\psi}^{\widehat{A}}(X)\right]=p_{S}^{\mathrm{d}}(F) \operatorname{Tr}\left[\rho_{\psi} P^{\widehat{A}}(X)\right],
$$

откуда вытекает условие, которому должна удовлетворять мера $p_{S}^{\mathrm{d}}(F)$.

\section{3. СОБСТВЕННЫЕ СМЕСИ В МОДЕЛИ РСР}

В силу приведенного в разделе 2 предположения вероятность $p_{S}(F)$ в соотношении (3) можно вычислить с применением стандартных правил KM, если $S$ представляет собой чистое состояние. Но при выводе формулы (3) не делалось никаких

\footnotetext{
6) Заметим, что представление обобщенной наблюдаемой, задаваемой в (5), индуцирует представление макроскопических свойств. Действительно, каждому $F=\left(A_{0}, X\right) \in \mathcal{F}_{0}$ можно поставить в соответствие семейство $\left\{T_{\psi}^{\widehat{A}}(X)\right\}_{|\psi\rangle \in \mathcal{V}}$ ограниченных положительно-определенных операторов (эффекты) в модели РСР.
} 
предположений относительно $S$, а потому оно должно оставаться справедливым и в случае, когда $S$ представляет собой собственную смесь (поскольку в данной работе рассматриваются только чистые смеси, то все смеси, рассматриваемые ниже, подразумеваются собственными). Можно задаться вопросом о том, можно ли вычислить $p_{S}(F)$ с помощью стандартных квантовых правил также и в данном случае. Авторами было недавно доказано, что ответ на этот вопрос отрицателен. Это привело к формулировке новых правил вычисления $p_{S}(F)$ и $p_{S}^{\mathrm{t}}(F)$ в случае смесей с применением математических подходов, обсуждавшихся в разделе 2 [32], [33]. В настоящей работе представлена усовершенствованная формулировка нашего подхода, и мы ограничимся рассмотрением только тех результатов, которые будут полезны в дальнейшем.

Пусть $S$ - смесь чистых состояний $S_{1}, S_{2}, \ldots$, заданных операторами плотности $\rho_{\psi_{1}}, \rho_{\psi_{2}}, \ldots$, с соответствующими вероятностями $p_{1}, p_{2}, \ldots$ Вероятность $p_{S}^{\mathrm{t}}\left(\left(A_{0}, X\right)\right)$ того, что измерение обобщенной наблюдаемой $A_{0}$ на физическом объекте $x$ в состоянии $S$ приводит к исходу, лежащему в борелевском множестве, $X \in \mathbb{B}(\mathbb{R})$, с $a_{0} \notin X$, или, эквивалентно, вероятность $p_{S}^{\mathrm{t}}(F)$ того, что $x$ обладает макроскопическим свойством $F=\left(A_{0}, X\right) \in \mathcal{F}$ при измерении свойства $F$ на данном состоянии, задается выражением

$$
p_{S}^{\mathrm{t}}\left(\left(A_{0}, X\right)\right)=p_{S}^{\mathrm{t}}(F)=\sum_{j} p_{j} p_{S_{j}}^{\mathrm{t}}(F)=\sum_{j} p_{j} p_{S_{j}}^{\mathrm{d}}(F) p_{S_{j}}(F)
$$

в силу справедливости формулы (3), в которой $S$ заменяется на $S_{j}$. Символ $p_{S_{j}}^{\mathrm{t}}(F)$ в соотношении (11) обозначает полную вероятность того, что физический объект $x$ в чистом состоянии $S_{j}$ проявляет свойство $F$, когда на нем производится (идеальное) измерение свойства $F$, величина $p_{S_{j}}^{\mathrm{d}}(F)$ - это вероятность регистрации объекта $x$, а через $p_{S_{j}}(F)$ обозначена условная вероятность того, что объект $x$ будет обладать свойством $F$ при регистрации. Снова применяя формулу (3), получим

$$
p_{S}(F)=\sum_{j} p_{j} \frac{p_{S_{j}}^{\mathrm{d}}(F)}{p_{S}^{\mathrm{d}}(F)} p_{S_{j}}(F)
$$

Соотношение (12) интуитивно понятно. В самом деле, множитель $p_{j} \frac{p_{S_{j}}^{\mathrm{d}}(F)}{p_{S}^{\mathrm{d}}(F)}$ в силу теоремы Байеса можно интерпретировать как условную вероятность того, что объект $x$ находился в состоянии $S_{j}$, когда измерялось свойство $F$ и регистрировался объект $x$.

Применив математическое представление из раздела 2, можно переписать соотношение (12). Из приведенного предположения получим

$$
p_{S_{j}}(F)=\operatorname{Tr}\left[\rho_{\psi_{j}} P^{\widehat{A}}(X)\right]
$$

где $P^{\widehat{A}}$ - (спектральная) положительно-определенная мера, ассоциированная с самосопряженным оператором $\widehat{A}$, представляющим $\mathrm{KM-наблюдаемую} A$, из которой получается наблюдаемая $A_{0}$. Следовательно, имеем

$$
p_{S}(F)=\operatorname{Tr}\left[\rho_{S}(F) P^{\widehat{A}}(X)\right],
$$


где

$$
\rho_{S}(F)=\sum_{j} p_{j} \frac{p_{S_{j}}^{\mathrm{d}}(F)}{p_{S}^{\mathrm{d}}(F)} \rho_{\psi_{j}}
$$

Более того, сделав очевидное предположение о существовании разложения

$$
p_{S}^{\mathrm{d}}(F)=\sum_{j} p_{j} p_{S_{j}}^{\mathrm{d}}(F)
$$

и заметив, что из соотношения (10) (которое выполняется только для чистых состояний) следует, что

$$
p_{S_{j}}^{\mathrm{d}}(F)=\frac{\operatorname{Tr}\left[\rho_{\psi_{j}} T_{\psi_{j}}^{\widehat{A}}(X)\right]}{\operatorname{Tr}\left[\rho_{\psi_{j}} P^{\widehat{A}}(X)\right]},
$$

получим в силу (15) следующее соотношение:

$$
\rho_{S}(F)=\sum_{j} p_{j} \frac{\operatorname{Tr}\left[\rho_{\psi_{j}} T_{\psi_{j}}^{\widehat{A}}(X)\right]}{\operatorname{Tr}\left[\rho_{\psi_{j}} P^{\widehat{A}}(X)\right]} \rho_{\psi_{j}}\left(\sum_{j} p_{j} \frac{\operatorname{Tr}\left[\rho_{\psi_{j}} T_{\psi_{j}}^{\widehat{A}}(X)\right]}{\operatorname{Tr}\left[\rho_{\psi_{j}} P^{\widehat{A}}(X)\right]}\right)^{-1}
$$

Из формул (14) и (18) следует, что вероятности $p_{S}(F)$, вообще говоря, не совпадают с вероятностью, получаемой при применении стандартных правил KM, т. е. при вычислении $\operatorname{Tr}\left[\rho_{S} P^{\widehat{A}}(X)\right]$, где $\rho_{S}=\sum_{j} p_{j} \rho_{\psi_{j}}$ - оператор плотности, представляющий в соответствии с правилами КМ смесь $S$. Это можно интуитивно объяснить с помощью того обстоятельства, что ансамбль $\mathscr{E}_{S}$ физических объектов, приготовленных в состоянии $S$, можно разбить на подансамбли $\mathscr{E}_{S_{1}}, \mathscr{E}_{S_{2}}, \ldots$ физических объектов, приготовленных в соответствующих состояниях ${ }^{7)} S_{1}, S_{2}, \ldots$ Когда макроскопическое свойство $F$ измеряется на $\mathscr{E}_{S}$, при каждом $\mathscr{E}_{S_{j}}$ подансамбль $\mathscr{E}_{S_{j}}$ регистрируемых объектов зависит не только от $F$, но и от $S_{j}$, а потому в общем случае подансамбль $\mathscr{E}_{S}^{\mathrm{d}}$ всех регистрируемых объектов не доставляет исследователю подлинный образец ансамбля $\mathscr{E}_{S}$. При рассмотрении вероятности $p_{S}(F)$ состояние $S$ должно представляться оператором плотности $\rho_{S}(F)$, зависящим от $F$ и совпадающим с $\rho_{S}$ только в некоторых специальных случаях. В более общей формулировке состояние $S$ должно быть ассоциировано с семейством операторов плотности

$$
\left\{\rho_{S}(F)\right\}_{F \in \mathcal{F}}=\left\{\sum_{j} p_{j} \frac{p_{S_{j}}^{\mathrm{d}}(F)}{p_{S}^{\mathrm{d}}(F)} \rho_{\psi_{j}}\right\}_{F \in \mathcal{F}} .
$$

Это соотношение задает представление состояния $S$ в модели РСР. Если рассматривать чистые состояния как предельные случаи смесей, то это семейство сведется к постоянной $\left\{\rho_{S}\right\}_{F \in \mathcal{F}}$ в случае, когда состояние $S$ чистое, откуда вытекает, что для выражения (14) выполнено предположение из раздела 2. Но из (14) следует также и то, что это предположение нельзя рапространить на случай смесей. Это наблюдение приводит к значимым физическим следствиям, поскольку из него вытекает,

\footnotetext{
7) Здесь применяется эпистемическая интерпретация вероятностей $p_{1}, p_{2}, \ldots$, которая принимается не всеми исследователями основ КМ (см. раздел 1), но которую можно подтвердить в концептуальном методе модели РСР, как показано в разделе 4.
} 
что в случае смесей вероятностные предсказания модели РСР не совпадают с предсказаниями КМ. При этом с помощью подходящего эксперимента, по крайней мере в принципе, можно определить, какая из теорий оказывается правильной.

Вернемся к рассмотрению вероятности $p_{S}^{\mathrm{t}}(F)$. Применяя формулу $(7)$, получим

$$
p_{S_{j}}^{\mathrm{t}}(F)=\operatorname{Tr}\left[\rho_{\psi_{j}} T_{\psi_{j}}^{\widehat{A}}(X)\right]
$$

где

$$
T_{\psi_{j}}^{\widehat{A}}(X)=\int_{X} p_{\psi_{j}}^{\mathrm{d}}(\widehat{A}, \lambda) d P_{\lambda}^{\widehat{A}}
$$

в силу формулы (6). Поэтому из равенства (11) следует, что

$$
p_{S}^{\mathrm{t}}(F)=\operatorname{Tr}\left[\sum_{j} p_{j} \rho_{\psi_{j}} T_{\psi_{j}}^{\widehat{A}}(X)\right]
$$

откуда вытекает, что в общем случае вероятность $p_{S}^{\mathrm{t}}(F)$ не записывается в форме следа произведения двух операторов, один из которых представляет состояние $S$, а второй - свойство $F$. Однако применяя (10) и (15), из формулы (21) можно получить соотношение

$$
p_{S}^{\mathrm{t}}(F)=\operatorname{Tr}\left[\sum_{j} p_{j} \rho_{\psi_{j}} p_{S_{j}}^{\mathrm{d}}(F) P^{\widehat{A}}(X)\right]=p_{S}^{\mathrm{d}}(F) \operatorname{Tr}\left[\rho_{S}(F) P^{\widehat{A}}(X)\right],
$$

которое согласуется с разложением (3).

Напомним, что $F \in \mathcal{F}$. Поэтому остается лишь рассмотреть макроскопическое свойство $G=\left(A_{0}, Y\right) \in \mathcal{F}_{0} \backslash \mathcal{F}$. В этом случае с помощью несложных вычислений [32], [33] приходим к следующему результату:

$$
p_{S}^{\mathrm{t}}(G)=\operatorname{Tr}\left[\sum_{j} p_{j} \rho_{\psi_{j}} T_{\psi_{j}}^{\widehat{A}}(Y)\right]
$$

который совпадает с выражением (21) при замене $F$ на $G$.

\section{4. ОПЕРАЦИОНАЛЬНОЕ ОПРЕДЕЛЕНИЕ СМЕСЕЙ}

В этом разделе мы сравниваем метематическое представление (собственных) смесей в модели РСР с представлением КМ и показываем, что в первом представлении удается избежать ряда проблем, возникающих во втором представлении.

Начнем с описания типичной процедуры приготовления физического объекта $x$ в состоянии $S$, когда $S$ представляет собой смесь, введенную в разделе 3.

Выберем приготавливающее устройство $\pi_{j}$ для каждого чистого состояния $S_{j}$, применим $\pi_{j}$ для приготовления ансамбля $\mathscr{E}_{S_{j}}$, состоящего из $n_{j}$ физических объектов в состоянии $S_{j}$, и выберем $n_{j}$ такое, что $n_{j}=N p_{j}$, где $N=\sum_{j} n_{j}$. Затем смешаем ансамбли $\mathscr{E}_{S_{1}}, \mathscr{E}_{S_{2}}, \ldots$, получив в результате ансамбль $\mathscr{E}_{S}$, состоящий из $N$ физических объектов, и сотрем всю информацию о способе смешения ансамблей $\mathscr{E}_{S_{1}}, \mathscr{E}_{S_{2}}, \ldots$, после чего выберем физический объект $x$ в $\mathscr{E}_{S}$.

Из этого описания следует, что в пределе большого числа частиц часто́ты стремятся к вероятностям, которые могут быть интерпретированы как эпистемические, 
т. е. формализующие потерю информации относительно чистого состояния, в котором на самом деле был приготовлен каждый из физических объектов (интерпретация незнания). Также отсюда вытекает, что можно построить много процедур приготовления физического объекта в состоянии $S$, и потому это определение называется операциональным определением состояния $S$, а через $\sigma_{S}$ обозначается множество различных приготовляющих процедур состояния $S$, которые можно получить, выбирая приготавливающие устройства, находящиеся в состояниях $S_{1}, S_{2}, \ldots$, всеми возможными способами ${ }^{8)}$.

Обратимся теперь к КМ. В этой теории состояния определяются как классы вероятностно эквивалентных приготавливающих устройств [8], и каждый класс эквивалентности задается своим оператором плотности (теорема Глисона). Оператор плотности, представляющий смесь, можно в общем случае разложить различными способами в выпуклую комбинацию операторов плотности, представляющих чистые состояния (неоднозначная разложимость квантовых смесей [8]), что обычно принимается как отличительная черта KM. Но из этого свойства вытекают проблемы интерпретации, что было отмечено в разделе 1. Обсудим это обстоятельство более детально. Для этого рассмотрим введенную выше смесь $S$. Ее операциональное определение предполагает, что она представима в КМ в виде оператора плотности $\rho_{S}=\sum_{j} p_{j} \rho_{\psi_{j}}$, где $p_{1}, p_{2}, \ldots$ - эпистемические вероятности. Однако хорошо известно, что, вообще говоря, существуют операторы проекций $\rho_{\chi_{1}}, \rho_{\chi_{2}}, \ldots$ на одномерные пространства, причем ни один из этих операторов не совпадает ни с одним из проекционных операторов $\rho_{\psi_{1}}, \rho_{\psi_{2}}, \ldots$, которые таковы, что $\rho_{S}=\sum_{l} q_{l} \rho_{\chi_{l}}, 0 \leqslant q_{l} \leqslant 1$, и $\sum_{l} q_{l}=1$. Если принять за основу такое определение оператора $\rho_{S}$, то коэффициенты $q_{l}$ невозможно интерпретировать как вероятности в рамках интерпретации незнания, поскольку $\rho_{\chi_{l}}$ не является представителем никакого возможного чистого состояния физического объекта в состоянии $S$ (в самом деле, $q_{1}, q_{2}, \ldots$ получаются при этом как комбинации эпистемических вероятностей $p_{1}, p_{2}, \ldots$ с неэпистемическими квантовыми вероятностями, см. раздел 1$)$. Рассмотрим теперь смесь $T$ чистых состояний $T_{1}, T_{2}, \ldots$, представленных операторами плотности $\rho_{\chi_{1}}, \rho_{\chi_{2}}, \ldots$, с соответствующими вероятностями $q_{1}, q_{2}, \ldots$, изготовленных согласно процедуре, описанной в случае состояния $S$, с учетом очевидных изменений. Из этого следует, что смесь $T$ допускает операциональное определение $\sigma_{T}$, отличное от $\sigma_{S}$. Несмотря на эти различия, выбор вероятностей и состояний предполагает, что $\rho_{S}=\rho_{T}$, а потому $\sigma_{S}$ и $\sigma_{T}$ оказываются вероятностно эквивалентными, и состояние $T$ следует отождествить с состоянием $S$ в соответствии с $\mathrm{KM}$, поскольку оно задается тем же самым оператором плотности. Но вероятности $q_{1}, q_{2}, \ldots$ теперь допускают интерпретацию незнания, которая противоречит заключению, полученному при рассмотрении состояния $S$.

8) В стандартном подходе КМ типичная процедура, приготавливающая физический объект $x$ в состоянии $S_{j}$, состоит в выборе максимальной наблюдаемой $A$ такой, что $S_{j}$ оказывается собственным состоянием $A$, с последующим осуществлением идеального измерения, в котором $x$ регистрируется тогда и только тогда, когда исход измерения представляет собой собственное значение $a_{j}$ оператора $\widehat{A}$, ассоциированного с состоянием $S_{j}$. Мы также считаем такую процедуру действующей в нашем подходе (в котором отсутствует процедура объективизации, см. раздел 2), также принимая в расчет возможность исхода "отсутствие регистрации" $A_{0}$ как одного из возможных исходов модели РСР, вследствие чего возникает необходимость ОПП, приведенного в разделе 2. 
Ввиду этого обстоятельства многие исследователи полагают, что интерпретацию вероятностей с точки зрения незнания, появляющуюся в различных возможных выражениях для $\rho_{S}$, следует отбросить вместе с интерпретациями вероятностей $p_{j}$ и $q_{l}$, следующими из операциональных определений $\sigma_{S}$ и $\sigma_{T}$ (см. раздел 1$)$. Мы, напротив, разделяем позицию тех авторов, которые утверждают, что стандартное математическое представление смесей не учитывает некоторых физически зна́чимых различий (см. дискуссию в разделе 1). В самом деле, различные операциональные определения, которые оказываются вероятностно эквивалентными (например, $\sigma_{S}$ и $\left.\sigma_{T}\right)$, отвечают различным формальным выражениям для одного и того же оператора плотности $\left(\rho_{S}=\rho_{T}\right)$, а следовательно, и различным интерпретациям коэффициентов, возникающих в любом заданном разложении этого оператора $\left(p_{1}, p_{2}, \ldots\right.$ или $\left.q_{1}, q_{2}, \ldots\right)$. Но предположение, что оператор плотности описывает данное состояние, не делает выделенным ни одно из его разложений по чистым состояниям, а потому физическая информация теряется, если принять за основу математическое представление смеси в КМ, которое привносит описанную выше неоднозначность в интерпретацию коэффициентов разложения.

Подведем итог с помощью следующей коммутативной диаграммы, задающей описание в KM:

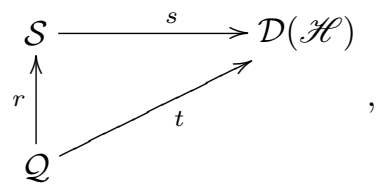

где $\mathcal{Q}$ - множество всех операциональных определений состояний, $\mathcal{S}$ - множество всех состояний, $\mathcal{D}(\mathscr{H})$ - выпуклое множество всех операторов плотности на $\mathscr{H}$. Отображения в диаграмме (24) определяются следующим образом:

$r: \sigma_{S} \in \mathcal{Q} \longmapsto S \in \mathcal{S}$ отображает каждый класс вероятностно эквивалентных операциональных определений в некоторое состояние;

$s: S \in \mathcal{S} \longmapsto \rho_{S} \in \mathcal{D}(\mathscr{H})$ - стандартное представление состояния $\mathcal{S} ;$

$t: \sigma_{S} \in \mathcal{Q} \longmapsto \rho_{S} \in \mathcal{D}(\mathscr{H})$ отображает каждый класс вероятностно эквивалентных операциональных определений в оператор плотности.

В силу замечаний, сделанных выше, отображение $s$ взаимно однозначно, в то время как отображения $r$ и $t$ сюръективны, но не взаимно однозначны (хотя их ограничения на операциональные определения только для чистых состояний, очевидно, взаимно однозначны).

Рассмотрим теперь модель РСР. В ней присутствует объективность свойств, а потому все вероятности оказываются эпистемическими, в силу чего удается с самого начала избежать возникновения проблемы интерпретации, вызванной смешением эпистемических и неэпистемических вероятностей. Более того, в математическом представлении смесей отсутствует явление потери физической информации. Кроме того, в модели РСР состояние определяется как класс вероятностно эквивалентных приготавливающих устройств (см. раздел 2), и может случиться, что два различных операциональных определения смесей, например $\sigma_{S}$ и $\sigma_{T}$, окажутся вероятностно эквивалентными $\left(\sigma_{S} \equiv \sigma_{T}\right.$, откуда следует, что эти состояния были составлены из вероятностно эквивалентных приготавливающих устройств), а смеси $S$ и $T$, операционально определяемые соответствующими операторами $\sigma_{S}$ и $\sigma_{T}$, следует отождествить, если только $\sigma_{S} \equiv \sigma_{T}$. Но в модели РСР семейства операторов плотности, ассоциированных с $S$ и $T$ (см. (19)), составлены при обращении к соответствующим 
операторам $\sigma_{S}$ и $\sigma_{T}$ и не обязательно совпадают. Итог можно представить в виде коммутативной диаграммы

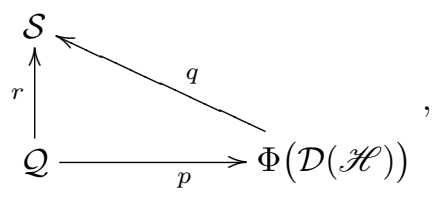

где символами $\mathcal{Q}$ и $\mathcal{S}$ обозначены те же величины, что и на диаграмме $(24), \Phi(\mathcal{D}(\mathscr{H}))$ множество всех семейств вида $\left\{\rho_{S}(F)\right\}_{F \in \mathcal{F}}$ с $S \in \mathcal{S}$, а сюръективные отображения $p, q, r$ определяются следующим образом:

$$
\begin{aligned}
& p: \sigma_{S} \in \mathcal{Q} \longmapsto\left\{\rho_{S}(F)\right\}_{F \in \mathcal{F}} \in \Phi(\mathcal{D}(\mathscr{H})) ; \\
& q:\left\{\rho_{S}(F)\right\}_{F \in \mathcal{F}} \in \Phi(\mathcal{D}(\mathscr{H})) \longmapsto S \in \mathcal{S} ; \\
& r: \sigma_{S} \in \mathcal{Q} \longmapsto S \in \mathcal{S} .
\end{aligned}
$$

Сделаем дополнительно предположение, что из утверждения $\sigma_{S} \neq \sigma_{T}$ следует утверждение $\left\{\rho_{S}(F)\right\}_{F \in \mathcal{F}} \neq\left\{\rho_{T}(F)\right\}_{F \in \mathcal{F}}$, которое физически оправданно, поскольку семейства $\left\{\rho_{S}(F)\right\}_{F \in \mathcal{F}}$ и $\left\{\rho_{T}(F)\right\}_{F \in \mathcal{F}}$ были построены в разделе 3 на основе соответствующих операциональных определений $\sigma_{S}$ и $\sigma_{T}$. Тогда отображение $p$ оказывается взаимно однозначным, и, сопоставляя смеси ее математическое представление, мы вычленим ее операциональное определение без потери физической информации, как указывалось выше.

На основе диаграммы (25) можно сделать важное теоретическое предположение. В самом деле, даже если отображение $p$ взаимно однозначно, отображения $q$ и $r$ не являются таковыми, что предполагает, что класс приготавливающих устройств, характеризующий смесь $S$, можно разбить на подклассы, отвечающие различным операциональным определениям состояния $S$. Отсюда можно заключить, что стандартные соотношения эквивалентности, задающие состояния на основе вероятностных соображений, необходимо подправить с тем, чтобы принять в расчет некоторые физически важные операциональные различия (см. также работу [19]).

\section{5. ОБОБЩЕННЫЙ ПОСТУЛАТ ЛЮДЕРСА}

В разделе 2 было показано, что ОПП задает преобразование чистого состояния, задаваемое процессом идеального неразрушающего измерения. Авторами недавно было показано, что с помощью результатов, приведенных в разделе 3 , и ОПП удается предсказать вид преобразования состояния, вызванного измерением того же типа, если состояние измеряемого объекта оказывается смешанным (обобщенный постулат Людерса). Для единства изложения приведем здесь этот результат [32], [33].

ОБОБЩЕННЫЙ ПОСТУЛАТ ЛЮДЕРСА. Пусть $S$ nредставляет собой смесъ чистых состояний $S_{1}, S_{2}, \ldots$, представленных операторами плотности $\rho_{\psi_{1}}, \rho_{\psi_{2}}, \ldots$ с соответствующими вероятностями $p_{1}, p_{2}, \ldots$, и пусть над физическим обгектом $x$, находящимся в состоянии $S$, производится неразрушающее идеальное измерение макроскопического свойства $F=\left(A_{0}, X\right) \in \mathcal{F}_{0}$.

Пусть итогом измерения оказывается положительный ответ. Тогда состояние $S_{F}$ обгекта $x$ после произведения регистрации есть смесь чистых состояний $S_{1 F}, S_{2 F}, \ldots$, представляемая соответствуюшими операторами плотности 
$\rho_{\psi_{1 F}}, \rho_{\psi_{2 F}}, \ldots$ такими, что для каждого $j=1,2, \ldots$

$$
\rho_{\psi_{j F}}=\frac{T_{\psi_{j}}^{\widehat{A}}(X) \rho_{\psi_{j}} T_{\psi_{j}}^{\widehat{A} \dagger}(X)}{\operatorname{Tr}\left[T_{\psi_{j}}^{\widehat{A}}(X) \rho_{\psi_{j}} T_{\psi_{j}}^{\widehat{A} \dagger}(X)\right]},
$$

и соответствующими вероятностями $p_{1 F}, p_{2 F}, \ldots$ такими, что

$$
p_{j F}=p_{j} \frac{p_{S_{j}}^{\mathrm{t}}\left(\left(A_{0}, X\right)\right)}{p_{S}^{\mathrm{t}}\left(\left(A_{0}, X\right)\right)}=p_{j} \frac{\operatorname{Tr}\left[\rho_{\psi_{j}} T_{\psi_{j}}^{\widehat{A}}(X)\right]}{\operatorname{Tr}\left[\sum_{j} p_{j} \rho_{\psi_{j}} T_{\psi_{j}}^{\widehat{A}}(X)\right]},
$$

a потому $S_{F}$ представляется семейством операторов плотности

$$
\left\{\rho_{S_{F}}(H)\right\}_{H \in \mathcal{F}}=\left\{\sum_{j} p_{j F} \frac{p_{S_{j F}}^{\mathrm{d}}(H)}{p_{S_{F}}^{\mathrm{d}}(H)} \rho_{\psi_{j F}}\right\}_{H \in \mathcal{F}} .
$$

Пусть итог измерения отричателен. Тогда состояние $S_{F}^{\prime}$ объекта х после произведения регистрации есть смесь чистых состояний $S_{1 F}^{\prime}, S_{2 F}^{\prime}, \ldots$, представленных соответствующими операторами плотности $\rho_{\psi_{1 F}^{\prime}}^{\prime}, \rho_{\psi_{2 F}^{\prime}}, \ldots$ такими, что для каждого $j=1,2, \ldots$

$$
\rho_{\psi_{j F}^{\prime}}=\frac{T_{\psi_{j}}^{\widehat{A}}(\mathbb{R} \backslash X) \rho_{\psi_{j}} T_{\psi_{j}}^{\widehat{A} \dagger}(\mathbb{R} \backslash X)}{\operatorname{Tr}\left[T_{\psi_{j}}^{\widehat{A}}(\mathbb{R} \backslash X) \rho_{\psi_{j}} T_{\psi_{j}}^{\widehat{A} \dagger}(\mathbb{R} \backslash X)\right]},
$$

и соответствующими вероятностями $p_{1 F}^{\prime}, p_{2 F}^{\prime}, \ldots$ такими, что

$$
p_{j F}^{\prime}=p_{j} \frac{p_{S_{j}}^{\mathrm{t}}\left(\left(A_{0}, \mathbb{R} \backslash X\right)\right)}{p_{S}^{\mathrm{t}}\left(\left(A_{0}, \mathbb{R} \backslash X\right)\right)}=p_{j} \frac{\operatorname{Tr}\left[\rho_{\psi_{j}} T_{\psi_{j}}^{\widehat{A}}(\mathbb{R} \backslash X)\right]}{\operatorname{Tr}\left[\sum_{j} p_{j} \rho_{\psi_{j}} T_{\psi_{j}}^{\widehat{A}}(\mathbb{R} \backslash X)\right]},
$$

a потому $S_{F}^{\prime}$ представляется семейством операторов плотности

$$
\left\{\rho_{S_{F}^{\prime}}(H)\right\}_{H \in \mathcal{F}}=\left\{\sum_{j} p_{j F}^{\prime} \frac{p_{S_{j F}^{\prime}}^{\mathrm{d}}(H)}{p_{S_{F}^{\prime}}^{\mathrm{d}}(H)} \rho_{\psi_{j F}^{\prime}}\right\}_{H \in \mathcal{F}} .
$$

Ясно, что обобщенный постулат Людерса является обобщением ОПП, поскольку выражения (26) и (29) совпадают с соответствующими формулами (8) и (9) в случае, когда $S$ представляет собой чистое состояние.

\section{Список литературы}

[1] P. Busch, P. J. Lahti, P. Mittelstaedt, The Quantum Theory of Measurement, Lecture Notes in Physics, 2, Springer, Berlin, 1991.

[2] P. Busch, A. Shimony, Stud. Hist. Phil. Mod. Phys., 27:4 (1996), 397-404, arXiv: quant-ph/9604013.

[3] P. Busch, Int. J. Theor. Phys., 37 (1998), 241-247, arXiv: quant-ph/9802011.

[4] И. Нейнман, Математические основы квантовой механики, Наука, М., 1964.

[5] H. Putnam, "A philosopher looks at quantum mechanics", Beyond the Edge of Certainty: Essays in Contemporary Science and Philosophy, ed. R. G. Colodny, Prentice-Hall, Englewood Cliffs, NJ, 1965, 75-101. 
[6] U. Fano, Rev. Mod. Phys., 29 (1957), 74-93.

[7] J. L. Park, Phil. Sci., 35:3 (1968), 205-231; 35:4, 389-411.

[8] E. G. Beltrametti, G. Cassinelli, The Logic of Quantum Mechanics, Encyclopedia Math. Appl., 15, Addison-Wesley, Reading, MA, 1981.

[9] L. E. Ballentine, Quantum Mechanics. A Modern Development, World Scientific, Singapore, 1998.

[10] B. d'Espagnat, Conceptual Foundations of Quantum Mechanics, Math. Phys. Monogr. Ser., 20, Benjamin, Reading, MA, London, Amsterdam, 1976.

[11] C. G. Timpson, H. R. Brown, Int. J. Quantum Inf., 3:4 (2005), 679-690.

[12] J.S. Bell, Physics, 1 (1964), 195-200.

[13] J. S. Bell, Rev. Mod. Phys., 38:3 (1966), 447-452.

[14] S. Kochen, E. P. Specker, J. Math. Mech., 17 (1967), 59-87.

[15] C. Garola, L. Solombrino, Found. Phys., 26:9 (1996), 1121-1164.

[16] C. Garola, L. Solombrino, Found. Phys., 26:10 (1996), 1329-1356.

[17] C. Garola, Found. Phys., 30:9 (2000), 1539-1565.

[18] C. Garola, "An epistemological criticism to the Bell-Kochen-Specker theorem", Foundations of Probability and Physics - 5 (Växjö, Sweden, 24-27 August 2008), AIP Conf. Proc., 1101, eds. L. Accardi et al., AIP, Melville, NY, 2009, 51-52.

[19] К. Гарола, С. Соццо, ТМФ, 152:2 (2007), 248-264; arXiv: quant-ph/0611072.

[20] Ф. Масилло, Дж. Сколаричи, С. Соццо, ТМФ, 160:1 (2009), 157-167; arXiv: 0901.0795.

[21] C. Garola, Found. Phys., 32:10 (2002), 1597-1615.

[22] C. Garola, Found. Phys. Lett., 16:6 (2003), 605-612.

[23] C. Garola, J. Pykacz, Found. Phys., 34:3 (2004), 449-475, arXiv: quant-ph/0304025.

[24] C. Garola, "The ESR model: Reinterpreting quantum probabilities within a realistic and local framework", Quantum Theory: Reconsideration of Foundations - 4 (Växjö, Sweden, 11-16 June 2007), AIP Conf. Proc., 962, eds. G. Adenier et al., AIP, Melville, NY, 2007, $247-252$.

[25] S. Sozzo, "Modified BCHSH inequalities within the ESR model", Quantum Theory: Reconsideration of Foundations - 4 (Växjö, Sweden, 11-16 June 2007), AIP Conf. Proc., 962, eds. G. Adenier et al., AIP, Melville, NY, 2007, 334-338.

[26] C. Garola, "A proposal for embodying quantum mechanics in a noncontextual framework by reinterpreting quantum probabilities", Foundations of Probability and Physics - 5 (Växjö, Sweden, 24-27 August 2008), AIP Conf. Proc., 1101, eds. L. Accardi et al., AIP, Melville, NY, 2009, 42-50.

[27] C. Garola, S. Sozzo, Int. J. Theor. Phys., 49:12 (2010), 3101-3117, arXiv: 0811.0539.

[28] O. Nánásiová, A. Yu. Khrennikov, Int. J. Theor. Phys., 45:3 (2006), 481-494.

[29] S. Sozzo, "Discrete generalized observables and ideal measurements in the ESR model: A Hilbert space representation", Foundations of Probability and Physics - 5 (Växjö, Sweden, 24-27 August 2008), AIP Conf. Proc., 1101, eds. L. Accardi et al., AIP, Melville, NY, 2009, 381-385.

[30] C. Garola, S. Sozzo, Europhys. Lett., 86:2 (2009), 20009.

[31] S. Sozzo, C. Garola, Int. J. Theor. Phys., 49:12 (2010), 3262-3270.

[32] C. Garola, S. Sozzo, Found. Phys., 41:3 (2011), 424-449, arXiv: 1001.4688.

[33] C. Garola, S. Sozzo, "The representation of mixtures in the ESR model for QM", Quantum Theory: Reconsideration of Foundations - 5 (Växjö, Sweden, 14-18 June 2009), AIP Conf. Proc., 1232, eds. L. Accardi et al., AIP, Melville, NY, 2010, 58-71.

[34] C. Garola, S. Sozzo, Humana. Mente, 13 (2010), 81-101. 